\title{
Comparative Evaluation of Different Phenotypic Methods in Detection of Klebsiella pneumoniae Carbapenemase (KPC)
}

\author{
A. Tejashree* and M. Archana Hegde \\ Department of Microbiology, JSS Medical College, Mysore, Karnataka, India \\ *Corresponding author
}

\begin{tabular}{|l|}
\hline K e y w o r d s \\
Carbapenem \\
Resistant-K. \\
pneumoniae, Modified \\
Hodge test, Carba NP \\
test, HiChrome agar \\
KPC test.
\end{tabular}

\section{Introduction}

Klebsiella pneumoniae, a gram negative bacilli belonging to the family Enterobacteriaceae are ubiquitously present and reported worldwide ${ }^{1}$. In recent years, $K$. pneumoniae have become important pathogens in nosocomial infections ${ }^{2}$. An estimated $8 \%$ of all nosocomial bacterial infections such as septicemia, pneumonia and urinary tract infections are caused by Klebsiellae, mainly by Klebsiella pneumoniae and Klebsiella oxytoca, primarily affecting immuno compromised patients ${ }^{3,4} \cdot K$. pneumoniae strains that produce extended spectrum $\beta$-lactamase enzymes (ESBLs) with 
transferable resistance to all $\beta$-lactams (except cephamycins and carbapenems) were first detected in the mid-1980s in Western Europe $^{5}$. Currently, there is a worldwide and non-uniform spread of the ESBL-producing $K$. pneumoniae with a prevalence as high as $45.4 \%$ of all $K$. pneumoniaeisolates ${ }^{6}$. Carbapenem antibiotics are considered as the drugs of choice for the treatment of extended spectrum beta-lactamase (ESBL)-producing Enterobacteriaceae and other multidrug resistant bacteria. ${ }^{7}$ The emergence of bacterial strains that produce carbapenemases further limits the therapeutic options available to clinicians. However, resistance to carbapenems is being increasingly detected and is mainly related to the action of carbapenemase-type enzymes. Carbapenem resistant Klebsiella pneumoniae is becoming threat globally and also as nosocomial infection because of its resistance to all carbapenem group of antibiotics. This study will help in betterment of patients in ICUs and also help to control nosocomial infections and to know the rapid technique to detect carbapenem resistance.

\section{Materials and Methods}

This was a hospital based prospective study, undertaken in the department of Microbiology. All clinical isolates of Klebsiella pneumoniae were collected from December 2014 to November 2016 was included in the study. Fecal K. pneumoniae was excluded from this study and inclusion criteria were only Klebsiella pneumoniae subspecies pneumoniae. The samples collected were processed as per standard methods. The study protocol was approved by the ethics committee of the institute.

Blood, Urine, CSF, Sputum, Ear and Nose swab, Abscess, Pus, Wound swab aspirates, Tissue were received in the laboratory were subjected to routine processing as per standard operating procedures. Phenotypic Identification of $K$. pneumoniae was carried out on Mac-Conkey Agar with Pink, round, large, dome shaped, mucoid, Lactose fermenting colonies. Followed by Blood Agar showing Grey, round, dome shaped large colonies.Gram Stain was carried indicating Gram negative rod, thick, short, rod shaped, capsulated, non- motile, $1-2 \times 0.5-0.8 \mu \mathrm{m}$ arranged singly or in pairs. Colonies were later subjected to biochemical tests such as, catalase, oxidase, Indole production, Urease hydrolysis, utilization of citrate, Triple sugar Iron agar test, Methyl red test, VogesProskauer test as per standard CLSI guidelines ${ }^{8}$.Antimicrobial susceptibility testing was performed as per the CLSI guidelines (2013) by Vitek 2 automated system to detect carbapenem resistant $K$. pneumoniae (CRKP) as primary test. Further, confirmatory test was carried by Modified Hodge test, HiCrome Agar KPC test and Carba NP test as different phenotypic tests.

\section{Modified Hodge test}

Prepare a 0.5 McFarland dilution of the $E$. coli ATCC 25922 in $5 \mathrm{ml}$ of nutrient broth. Dilute $1: 10$ by adding $0.5 \mathrm{ml}$ of the 0.5 McFarland to $4.5 \mathrm{ml}$ of saline. Streak a lawn of the 1:10 dilution of E. coli ATCC 25922 on Mueller Hinton agar plate and allow to dry 35 minutes. A $10 \mu \mathrm{g}$ Ertapenem susceptibility disk (CT1761B-ETP 10mcg, [B. No.-178667] Oxoid, UK.) was placed in the center of the test area. In a straight line, test organism was streaked from the edge of the disk to the edge of the plate. Up to four organisms can be tested on the same plate with one drug. Incubate overnight at $35^{\circ} \mathrm{C} \pm 2{ }^{\circ} \mathrm{C}$ in ambient air for 16-24 hours. After 16-24 hrs of incubation, examine plate for a clover leaftype indentation at intersection of test organism and E. coli 25922, within zone of inhibition of carbapenem susceptibility disk. MHT Positive test: Clover leaf-like 
indentation of E. coli 25922 growing along test organism growth streak within disk diffusion zone. MHT Negative test: No growth of E. coli 25922 along test organism growth streak within disc diffusion zone.

\section{HiCrome Agar KPC Test: (HIMEDIA M1831-100G)}

Suspend $16.50 \mathrm{~g}$ in $500 \mathrm{ml}$ distilled water. Heated to boiling point to dissolve the medium completely. Sterilized by autoclaving at $15 \mathrm{lbs}$ pressure $\left(121^{\circ} \mathrm{C}\right)$ for 15 minutes. Cooled to $45-50^{\circ} \mathrm{C}$ and aseptically the rehydrated contents of one vial of HiChrome KPC selective supplement (FD279) was added. Mixed well and poured into sterile Perti-plates.

Once the media is solidified, media plates are kept for quality control check for 24 hours, if plates do not show any growth, then quality check is approved. Direct plating of the sample is carried out using inoculation loop and incubated for 18-24 hours. After incubation plates are observed for Metallic Blue Colonies of Klebsiella pneumoniae which is considered to be positive. Bacterial colonies grown other than Klebsiella pneumoniae will be indicated by color differenciation as: E. coli-Pink color, Pseudomonas- Cream color, Bacteria which are not resistant to carbapenems will be inhibited/ not grown on culture plates.

\section{Carba NP Test $^{9}$}

One calibrated loop $(10 \mu \mathrm{L})$ of the tested strain directly recovered from the antibiogram was resuspended in a Tris- $\mathrm{HCl} 20 \mathrm{mmol} / \mathrm{L}$ lysis buffer (S D Fine Chemical LimitedSDFCL [37119 K01]- C16A/1915/0409/13, Mumbai, India). Vortexed for 1 minute and further incubated at room temperature for 30 minutes. This bacterial suspension was centrifuged at $10,000 \times g$ at room temperature for 5 minutes. $30 \mu \mathrm{L}$ of the supernatant, corresponding to the enzymatic bacterial suspension, was mixed with $100 \mu \mathrm{L}$ of a $1-\mathrm{mL}$ solution made of $3 \mathrm{mg}$ of Imipenem and Cilastin injection IP (Lupinem, Himachal Pradesh, India.[B.No.- HZP6001]), pH 7.8, phenol red solution(Nice Chemicals(P) LTD[P40671]-B.No.-409119, Kochi, India), and $0.1 \mathrm{mmol} / \mathrm{L} \mathrm{ZnSO}_{4}$ (S D Fine Chemical Limited-SDFCL [40621 K05]C16A/2516/0801/08, Mumbai, India). The phenol red solution was prepared by mixing $2 \mathrm{~mL}$ of a phenol red (Lupinem, Himachal Pradesh, India [B.No.- HZP6001]) solution $0.5 \%(\mathrm{wt} / \mathrm{vol})$ with $16.6 \mathrm{~mL}$ of distilled water. The $\mathrm{pH}$ value was then adjusted to 7.8 by adding drops of $1 \mathrm{~N} \mathrm{NaOH}$. A mixture of the phenol red solution and the enzymatic suspension being tested was incubated at $37^{\circ} \mathrm{C}$ for a maximum of 2 hours and the results were interpreted.

The color of the vials turned from red to orange or yellow for tested strains that were producing carbapenemases, whereas vials corresponding to bacterial extracts of isolates that did not produce carbapenemase remained red. The color changed from red to yellow as early as 5-10 minutes after incubation for KPC producers began, 30 min-VIM, 15-60 min- MBL (NDM-1), 30-60 min - OXA-48 may be present. In most cases, incubation for 30 minutes is sufficient for obtaining a frank color change for carbapenemase producers.

\section{Results and Discussion}

A total of 1,539 Klebsiella pneumoniae was isolated from various clinical samples over a period of two years from December 2014 to November 2016. Out of 1,539 Klebsiella pneumoniae isolates collected, 254 (16.50\%) isolates were carbapenem resistant Klebsiella pneumoniae (CRKP) (resistant to Imipenem/ Ertapenem/Meropenem/Doripenem antibiotics) by Vitek 2 . 


\section{Modified Hodge Test (MHT) result}

Out of 254 carbapenem resistant Klebsiella pneumoniae (CRKP) isolates, 184(72.44\%) were positive with clover-leaf indentation for Modified Hodge Test (MHT) and 70(27.55\%) were negative without clover-leaf indentation respectively (Fig. 1).

\section{HiCrome KPC agar test result}

Among 254 carbapenem resistant Klebsiella pneumoniae (CRKP) isolates, 235(92.51\%) were positive (Metallic blue) for HiCrome KPC agar test and 19(7.48\%) were negative by HiChrome KPC agar test (Fig. 2).

\section{Carba NP test result}

Out of 254 carbapenem resistant Klebsiella pneumoniae (CRKP) isolates, 239(94.09\%) were positive (Color change within $2 \mathrm{hrs}$ of incubation from red to orange/yellow color) for Carba NP test and 15(5.90\%) were negative without color change (showing red color) (Fig. 3).

Among 254 CRKP isolates, 181(71.25\%) were True Positive in all the test like HiCrome KPC agar test, Modified Hodge Test and Carba NP Test and 15(5.90\%) were True Negatives.

The potential of Klebsiella pneumoniae Carbapenamase (KPC) production among gram negative bacteria along with the development of resistance to carbapenem antibiotics have made difficult and important problem in the treatment of infection.

Detection of KPC producing strains by phenotypic method allows fast, cost effective and accurate results that can prevent spread of this mechanism of resistance. Many different phenotypic methods for detection of KPC have been suggested. ${ }^{1}$
Reduced susceptibility to carbapenems are indirect indicators, on which rapid identification of KPC can be followed. ${ }^{2}$ All Klebsiella pneumoniae isolates were inoculated on Mac Conkey agar medium. Antibiotic susceptibility testing was performed and CRKP was detected by the Vitek 2. In our study, a total of 254(16.50\%) Klebsiella pneumoniae isolates were Carbapenem resistant from Vitek 2 result. The first commercially available chromogenic medium HiCrome agar KPC was designed for isolation of carbapenem-resistant (CR) gram negative bacteria. Carbapenem resistant Klebsiella pneumoniae (CRKP) were excellently detected by HiCrome agar KPC medium by color and morphological characters, allowing rapid differentiation of the bacterial colonies. HiCrome agar KPC showed sensitivity and specificity when related to PCR (gold standard) were 100\% and $98.4 \%$ respectively. ${ }^{3}$

In our study, from 254 CRKP isolates, 235(92.51\%) were positive (Metallic blue) for Crome agar KPC test and 19(7.48\%) were negative by KPC test. $35 \%$ positive were seen in the test performed by Zmirasamra et al., ${ }^{3}$ with metallic blue colony, which is showing less positive rate when compared to our result and Crome agar medium can also be used for fast and direct identification of CRKP gram negative pathogen from clinical isolates. ${ }^{3}$

To rapidly identify carbapenemase producers in Enterobacteriaceae, Patrice Nordmannet $a l .{ }^{9}$ developed the Carba NP test based on the colorimetric detection of hydrolysis of the $\beta$ lactam ring of a carbapenem molecule. The test is based on in vitro hydrolysis of a carbapenem, ertapenem /imipenem and uses isolated bacterial colonies for identification. It was $100 \%$ sensitive and specific compared with molecular-based techniques. This rapid ( $<2$ hours), inexpensive technique may be implemented in any laboratory. 
Fig.1 Modified Hogde test

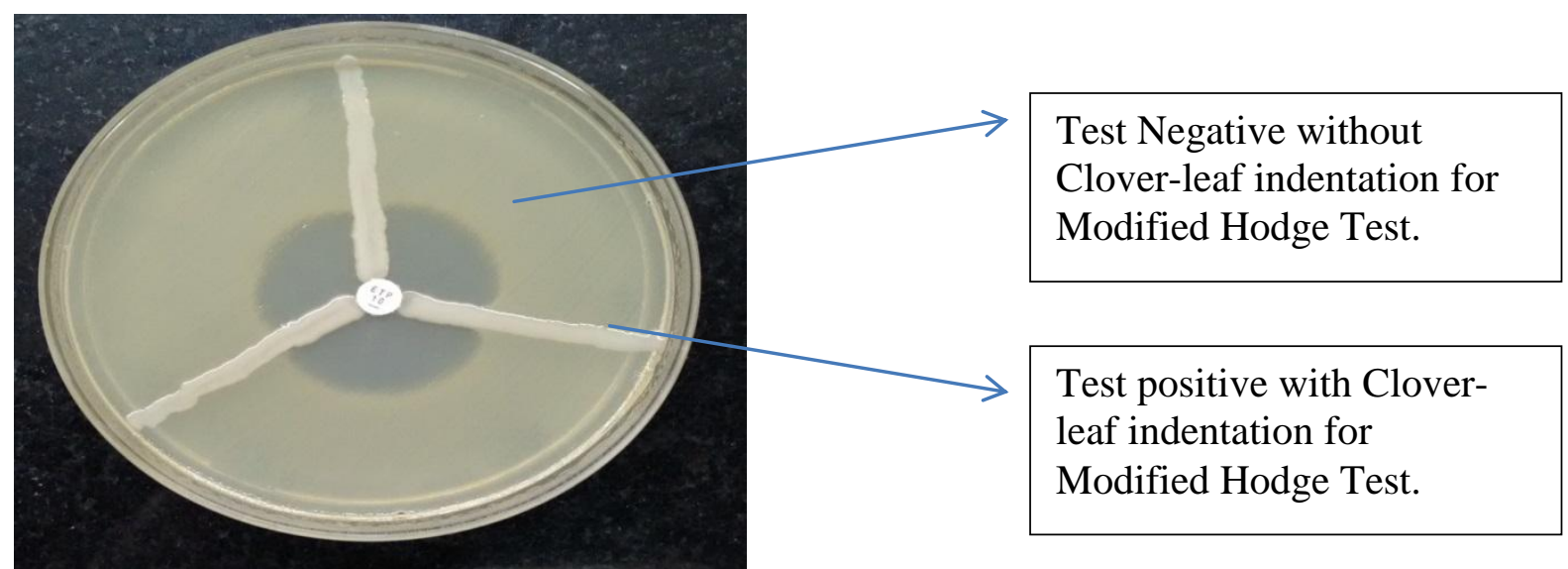

Fig.2 HiChrome agar KPC test

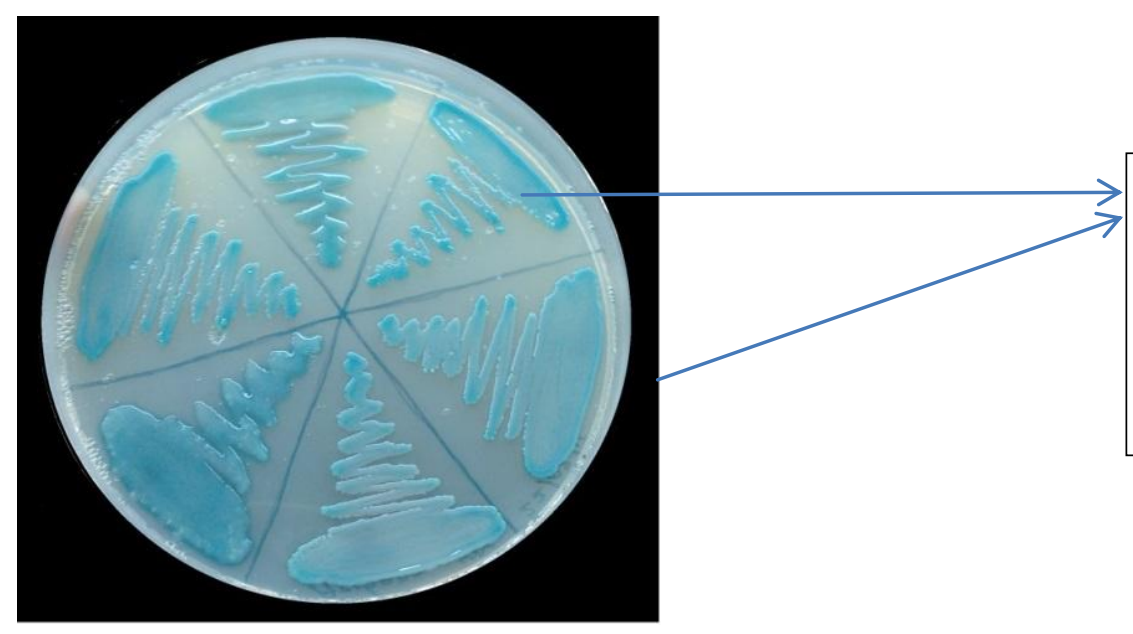

Test sample showing Metallic blue Carbapenem resistant Klebsiella pneumoniae colonies. Positive test for HiChrome KPC agar.

Fig.3 Carba NP test

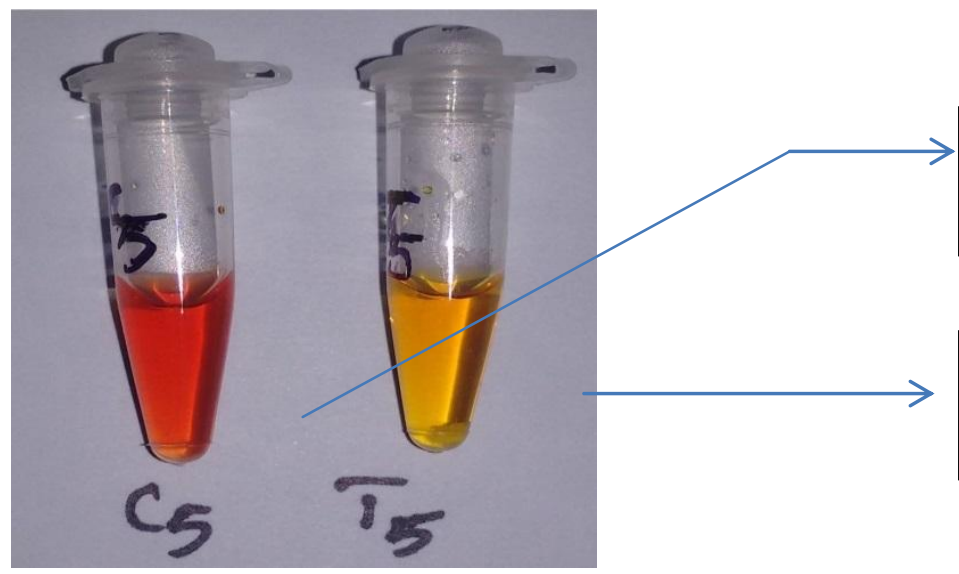

Control tube showing negative result with Red colour change for Carba NP Test

Test tube showing Positive result with Yellow colour change for Carba NP Test 
Table.1 Comparison of HiCrome KPC agar test, Modified Hodge test and Carba NP test result

\begin{tabular}{|c|c|c|c|c|c|c|}
\hline & KPC-P & KPC-N & MHT-P & MHT-N & CNP-P & CNP-N \\
\hline KPC-P & $235(92.51 \%)$ & - & $177(69.68 \%)$ & $58(22.83 \%)$ & 234(92.12\%) & $01(0.39 \%)$ \\
\hline KPC-N & - & $19(7.48 \%)$ & $04(1.57 \%)$ & $15(5.90 \%)$ & $05(1.96 \%)$ & $14(5.51 \%)$ \\
\hline MHT-P & $180(70.86 \%)$ & $04(1.57 \%)$ & $184(72.44 \%)$ & - & $184(72.44 \%)$ & $00(0.0 \%)$ \\
\hline MHT-N & $55(21.65 \%)$ & $15(5.90 \%)$ & - & $70(27.55 \%)$ & $55(21.65 \%)$ & $15(5.90 \%)$ \\
\hline CNP-P & $234(92.12 \%)$ & $05(1.96 \%)$ & 183(72.04\%) & $56(22.04 \%)$ & $239(94.09 \%)$ & - \\
\hline CNP-N & $01(0.39 \%)$ & $14(5.51 \%)$ & $00(0.0 \%)$ & $15(5.90 \%)$ & - & $15(5.90 \%)$ \\
\hline
\end{tabular}

In the current study, 254 CRKP isolates were tested for Carba NP test. Test result showed, 239 $(94.09 \%)$ were positive (Color change within $<2$ hours of incubation from red to orange/yellow color) for Carba NP test and $15(5.90 \%)$ were negative without color change (showing red color). Our results were similar to the result of Patrice Nordmann et $a l .{ }^{9}$. The strains give non interpretable results when bacterial colonies recovered from Drigalski lactose agar or MacConkey agar, regardless of carbapenemase production. Such results might be a consequence of the accumulation of lactic acid in the bacterial isolates that were able to ferment lactose. Therefore, the Carba NP test is not suitable to identify carbapenemase producers grown on Drigalski lactose agar and MacConkey agar plates. $^{10}$

In the present study, 254 CRKP were further tested for KPC and MBL production by Modified Hodge Test (MHT). Recently CLSI accepted MHT as specific and sensitive phenotypic method for carbapenemase detection $^{4,5}$. Out of 254 carbapenem resistant Klebsiella pneumoniae (CRKP) isolates, $190(74.80 \%)$ were positive with clover-leaf indentation for Modified Hodge Test (MHT) and 64(25.19\%) were negative without clover-leaf indentation respectively. Our results are similar with many other studies like, $75 \%$ positive result showed by McGettigan et al., ${ }^{6}$ followed $69 \%$ by Amjad et al., ${ }^{11}$, Cury et al.,(MHT $71 \%$ positive) ${ }^{4}$ and little higher rate was seen in Galani et al., with $98 \%{ }^{12}$, respectively.

In the current study, we have got $181(71.25 \%)$ true positives and $15(5.90 \%)$ true negative result for Crome agar KPC test, Modified Hodge Test and Carba NP test. This was similar to the study of Vanessa BleyRibeiro et al., with $70 \%$ true positives and showing slightly high, true negative results of $29 \%$. KPC carbapenemsase alone were seen, which may not be applied for other types of carbapenemase, hence MHT is not considered to be specific for KPC detection and sensitivity and specificity may differ in the similar way with other carbapenemases. With minimum carbapenemase activity, MHT initially aims to recognize and differentiate the presence of carbapenemase production with that of other enzymes. ${ }^{13}$ The Carba NP test has many advantages. It is not expensive, fast, can make multiple copies, and quick to detect and specific. It completely removes the need for using other proficiency to identify carbapenemase producers that are longdelayed and less receptive or specific. Using this distinct test would enhance the detection of patients infected or colonized with carbapenemase producers. ${ }^{9}$ 
In our study; out of 235 Crome agar KPC Test positive isolates; $234(92.12 \%)$ were positive for Carba NP test, which was similar to the result of HiCrome agar KPC test positive isolates and $01(0.39 \%)$ were negative for Carba NP test. Were as, 177 (69.68\%) isolates were found to produce carbapenemase enzyme by MHT and 58(22.83\%) were found negative for MHT. The reason for negative result of bacterial strains which were carbapenem resistant but negative by MHT could be explained as the overproduction of ESBL or Amp C enzyme with porin loss ${ }^{14}$. The processing of MHT showed that it is easy, simple with minimum infrastructure and of less cost, reliable test and this can be implemented in routine microbiology laboratories for detection of carbapenemase producers. One isolate (Sample No.-02) was negative by Carba NP Test and Modified Hodge Test but was positive by HiChrome KPC agar test. This isolate will be tested further for the presence of KPC gene by Molecular methods (PCR).

MHT was not specific for production of KPC as per Anderson et al., ${ }^{15}$; where as in other study by Girlich et al., ${ }^{16}$ showed $77.4 \%$ sensitivity and $38.9 \%$ specificity for MHT test in detecting KPC in overall test. This low sensitivity and specificity may be because, MHT detects other carbapenemase enzymes in addition to KPC as it is the only indicative enzymatic activity of carbapenemase and cannot differentiate class A carbapenemases from class B MBLs. And MHT cannot be used as a confirmatory test for recognition of the KPCs because of the difficult elucidation and false positive results. False-positive results are more common in isolates producing AmpC and CTX-M blactamase. $^{17,18,19 .}$

Isolates with reduced susceptibility to one or more carbapenems undergo further testing with the Modified Hodge Test (MHT), in order to detect the presence of a carbapenemase enzyme as recommended by $\mathrm{CDC}^{20,9}$. And the testing of MHT is not necessary, when the isolate is found to be intermediate or resistant to all carbapenems tested by CLSI guidelines, since use of the recently reduced breakpoints should preclude the possibility of misclassification of CR-KP as carbapenem susceptible. They do, however, suggest its use for epidemiological investigation. $^{21,9}$

In our study, out of $184(72.44 \%)$ MHT positive isolates; $4(1.57 \%)$ isolates were found Crome agar KPC test negative (with no metallic blue colony). This may be because, the remaining MHT positive isolates which were negative for KPCs and MBLs presumably had the other types of carbapenemase enzymes as the oxacillinase enzyme, as the OXA encoding gene has been found to be highly disseminated in the last few years. Not only do KPC-producing organisms hydrolyze carbapenems, but also, they are often resistant to multiple other antibiotics ${ }^{22}$. But, all the $184(72.44 \%)$ MHT positive isolates were seen positive for Crome agar KPC test (Table 1).

In the current study, among 254 CRKP isolates, 239(94.09\%) Carba NP test positive isolates, 234(92.12\%) were positive for Crome agar KPC test and 183(72.04\%) were positive for Modified Hodge Test. And $15(5.90 \%)$ isolates showed negative result for Carba NP test out of 254 CRKP isolates. This was very much similar to the result of Crome agar KPC with 14(5.51\%) negative test isolates and Modified Hodge Test showing $15(5.90 \%)$ negative result.

Resistance to carbapenems is being increasingly detected and is mainly related to the action of carbapenemase-type enzymes and becoming threat globally and also as nosocomial infection because of its resistance 
to all carbapenem group of antibiotics. In our study, $254 \quad(16.50 \%)$ isolates were carbapenem resistant Klebsiella pneumoniae (CRKP). Among the different phenotypic tests conducted, Carba NP test showed 239 (94.09\%) were positive (Color change within $2 \mathrm{hrs}$ of incubation from red to orange/yellow color), which wasrapid $(<2$ hours $)$, inexpensive technique compared to HiChrome agar KPC test and Modified hodge test. This technique can be implemented in the Microbiology laboratory for early detection of Carbapenem resistance (CR).Further, the test results need to be compared with Molecular results, as PCR is gold standard for detection of Carbapenem resistance and genes (KPC's and MBL types) coding for carbapenem resistance can be detected.

\section{References}

1. Gomez SA, Pasteran FG, Faccone D, Tijet N, Rapoport M, Lucero C, Lastovetska O, Albornoz E, Galas M; KPC Group, Melano RG, Corso A, Petroni A. Clonal dissemination of Klebsiella pneumoniae ST258 harbouring KPC-2 in Argentina. Clin. Microbiol. Infect. 2011 Oct; 17(10): 1520-4.

2. Smith Moland E, Hanson ND, Herrera VL, Black JA, Lockhart TJ, Hossain A, Johnson JA, Goering RV, Thomson KS. Plasmid-mediated, carbapenemhydrolysing beta-lactamase, KPC-2, in Klebsiella pneumoniae isolates. J Antimicrob. Chemother. 2003 Mar; 51(3): 711-4.

3. Samra Z, Bahar J, Madar-Shapiro L, Aziz N, Israel S, Bishara J. Evaluation of CHROMagar KPC for rapid detection of carbapenem-resistant Enterobacteriaceae. J ClinMicrobiol. 2008 Sep; 46(9): 3110-1.
4. Cury AP, Andreazzi D, Maffucci M, Caiaffa-Junior HH, Rossi F. The modified Hodge test is a useful tool for ruling out Klebsiella pneumoniae carbapenemase. Clinics (Sao Paulo). 2012 Dec; 67(12): 1427-31.

5. Chen $\mathrm{S}, \mathrm{Hu} \mathrm{F}, \mathrm{Xu} \mathrm{X}$, Liu Y, Wu W, Zhu D, et al., High Prevalence of KPC2-Type Carbapenemase Coupled with CTX-M-Type Extended-SpectrumLactamases in Carbapenem-Resistant Klebsiella pneumoniae in a Teaching Hospital in China. Antimicrob Agents Chemother, 2011 (5): 2493-4.

6. McGettigan SE, Andreacchio K, Edelstein PH. Specificity of ertapenem susceptibility screening for detection of Klebsiella pneumoniae carbapenemases. J ClinMicrobiol. 2009 Mar; 47(3): 7856.

7. Livermore DM. Has the era of untreatable infections arrived? J Antimicrob. Chemother. 2009 Sep; 64(Suppl 1): 29-36.

8. Clinical Laboratory Standards Institutes. Performance Standards for antimicrobial susceptibility testing, XVI International Supplement (M100-S16). Wayne, Pennsylvania, USA: National Committee for Clinical Laboratory Standards 2013.

9. Patrice Nordmann, Laurent Poirel, and Laurent Dortet, Rapid Detection of Carbapenemase producing Enterobacteriaceae, Emerging Infectious Diseases.

10. Dortet L, Bréchard L, Poirel L, Nordmann P. Impact of the isolation medium for detection of carbapenemase-producing Enterobacteriaceae using an updated version of the Carba NP test. J Med Microbiol. 2014 May; 63(Pt 5): 772-6.

11. Amjad A, MirzaIa, Abbasi S, Farwa U, Malik N, Zia F. Modified Hodge test: A simple and effective test for detection of 
carbapenemase production. Iran $\mathbf{J}$ Microbiol. 2011 Dec; 3(4):189-93.

12. Galani I, Rekatsina PD, Hatzaki D, Plachouras D, Souli M, Giamarellou H. Evaluation of different laboratory tests for the detection of metallo-betalactamase production in Enterobacteriaceae. J Antimicrob Chemother. 2008 Mar; 61(3): 548-53.

13. Ribeiro VB, Linhares AR, Zavascki AP, Barth AL. Performance of quantification of Modified Hodge Test: an evaluation with Klebsiella pneumoniae carbapenemase-producing Enterobacteriaceae isolates. Biomed Res Int. 2014; 2014: 139305.

14. Anderson KF, Lonsway DR, Rasheed JK, Biddle J, Jensen B, McDougal LK, Carey RB, Thompson A, Stocker S, Limbago B, Patel JB. Evaluation of methods to identify the Klebsiella pneumoniae carbapenemase in Enterobacteriaceae. J ClinMicrobiol. 2007 Aug; 45(8): 2723-5.

15. Girlich D, Poirel L, Nordmann P. Value of the modified Hodge test for detection of emerging carbapenemases in Enterobacteriaceae. J ClinMicrobiol. 2012 Feb; 50(2): 477-9.

16. Lee K, Chong Y, Shin HB, Kim YA, Yong D, Yum JH. Modified Hodge and
EDTA-disk synergy tests to screen metallo-beta-lactamase producing strains of Pseudomonas and Acinetobacter species. Clin Microbiol Infect. $2001 \mathrm{Feb}$; 7(2): 88-91.

17. Carvalhaes CG, Picão RC, Nicoletti AG, Xavier DE, Gales AC. Cloverleaf test(modified Hodge test) for detecting carbapenemase production in Klebsiella pneumoniae: be aware of false positive results. J Antimicrob. Chemother. 2010Feb; 65(2): 249-51.

18. Clinical and Laboratory Standards Institute: Performance Standards for Antimicrobial Susceptibility Testing; Twenty-Fourth Informational Supplement (January 2014). 2014.

19. Moran Gilad J, Carmeli Y, Schwartz D, Navon-Venezia S. Laboratory evaluation of the CHROM agar KPC medium for identification of carbapenem- nonsusceptible Enterobacteriaceae. Diagn Microbiol Infect Dis. 2011 Aug; 70(4): 565-7.

20. VanDuin D, Kaye KS, Neuner EA, Bonomo RA. Carbapenem-resistant Enterobacteriaceae: a review of treatment and outcomes. Diagn Microbiol Infect Dis. 2013 Feb; 75(2): 115-20.

\section{How to cite this article:}

Tejashree, A. and Archana Hegde, M. 2017. Comparative Evaluation of Different Phenotypic Methods in Detection of Klebsiella pneumoniae Carbapenemase (KPC). Int.J.Curr.Microbiol.App.Sci. 6(12): 2486-2494. doi: https://doi.org/10.20546/ijcmas.2017.612.289 